\title{
Sociología
}

Allain, M. (2019). Conflictos y protestas socioambientales en Chile: Reflexiones metodológicas y resultados. Revista de Sociología, 34(1), 81-101. doi: 10.5354/0719-529X.2019.54271

\section{Conflictos y protestas socio-ambientales en Chile: Reflexiones metodológicas y resultados}

\section{[Conflicts and socio-environmental protests in Chile: Methodological reflections and results]}

\author{
Mathilde Allain
}

Centro de Estudios de Conflicto y Cohesión Social

\begin{abstract}
Resumen
Este artículo presenta las primeras reflexiones basadas en el análisis de la base de datos del Observatorio de Conflictos del Centro de Estudios de Conflicto y Cohesión Social (COES). En una primera parte, el artículo detalla las principales bases de datos disponibles, aborda la definición de los términos "conflicto" y "socio-ambiental" y el rol clave de las investigaciones en visibilizar o invisibilizar procesos sociales. En una segunda parte se presentan algunos resultados de una investigación en curso que participa de la definición del "conflicto socioambiental" centrándose en uno de sus aspectos: la protesta. De esta forma se abordan dos preguntas centrales ¿Quiénes son los ciudadanos que protestan, y cuáles son sus demandas socio-ambientales? Este artículo puede ser útil para los estudiosos de los conflictos socio-ambientales confrontados a una variedad de fuentes de datos.
\end{abstract}

Palabras clave: conflictos socio-ambientales, metodologías mixtas, protestas, politización.

\begin{abstract}
This paper offers methodological discussion on today's available data on socio-environmental conflicts in Chile and presents first results based on an analysis of the Conflicts Observatory from the Centre for Social Conflicts and Cohesion Studies (COES). In the first part the article details the main database available and stress a discussion on the definition of the key terms "conflict" and "socio-environmental". That way the paper recall that investigations play a role in giving visibility or maintaining invisible social processes. In the second part, the paper presents an ongoing research results that participate to the definition of "socio-environmental conflicts" through the study of protests. Thus the article deals with two main questions: Who are the protesters, and what are their socio-environmental demands? This text can be useful for students and researchers working on socioenvironmental conflicts and dealing with a variety of data sources.
\end{abstract}

Keywords: socio-environmental conflicts, mix methods, protest, politicization.

Contacto: La comunicación sobre este artículo debe ser enviada a Mathilde Allain, email: mathilde.allain27@gmail.com

Financiamiento: Esta investigación tiene el apoyo financiero del Centro de Estudios de Conflicto y Cohesión Social (CONICYT/FONDAP/15130009).

Agradecimientos: La autora agradece los comentarios de Antoine Maillet, la colaboración, la calidad del trabajo de Pamela Gordillo y los intercambios fructíferos con el equipo del Fondecyt $\mathrm{N}^{\circ} 1151215$ "Lo que los conflictos producen" y Fondecyt $\mathrm{N}^{\circ} 1180496$ ¿De la dispersión a la acumulación? Consecuencias políticas de los conflictos socio-territoriales en perspectiva comparada (Chile y Perú)” (2018-2021). 


\section{INTRODUCCIÓN}

En Chile existen 116 conflictos socio-ambientales según el Instituto Nacional de Derechos Humanos (INDH, 2018); 51 casos de conflictos registrados según el Observatorio Latinoamericano de Conflictos Ambientales (OLCA, 2018); 48 conflictos mineros según el Observatorio de Conflictos Mineros de América Latina (OCMAL, s.f.); entre 5 y 7 "zonas de sacrificio", según las distintas ONGs que usan este término; 101 casos de conflictos socioterritoriales vinculados a las industrias minera y eléctrica entre 2005 y 2014 según el catastro del Proyecto Fondecyt "Lo que los conflictos producen" (Delamaza, Maillet, \& Martínez 2017 ; Maillet \& Albala, 2018) y 1,113 eventos de protestas socio-ambientales entre 2012 y 2017 según el Observatorio de Conflictos del Centro de Estudios de Conflicto y Cohesión Social (COES, 2018). Estas cifras responden a distintas lógicas políticas, militantes y científicas de registro de la conflictividad ligada a los temas socioambientales en el país. No tienen las mismas bases de estudio y tampoco, los mismos fundamentos, pero estos trabajos dan cuenta de un interés académico y social creciente por entender un fenómeno social, ambiental y político central en el Chile de hoy.

A la hora de ponerse a estudiar los conflictos socio-ambientales las investigadoras y los investigadores tienen la posibilidad de acceder a distintas fuentes de datos, sea para analizar y comparar casos específicos, testear hipótesis de trabajo o determinar un estudio de campo. Sin embargo, registrar, categorizar y determinar un conflicto como "socio-ambiental" o una situación como en "conflicto" no son procesos neutros, ya que contribuyen a visibilizar o invisibilizar procesos sociales complejos. Por consiguiente, es necesario entender las distintas fuentes de datos disponibles para generar información (acerca de los lugares, los territorios afectados, los ciudadanos movilizados, los proyectos problemáticos, los derechos en juego, las reivindicaciones expuestas, etc.), así como los procesos de construcción metodológica y conceptual de ellas. De acuerdo a quienes producen el conocimiento, los objetivos pueden variar: para algunos la meta es dar visibilidad a lo invisible para avanzar así una agenda política y/o social, mientras que para otros el objetivo es llegar a una mayor exhaustividad en la representación de lo que está ocurriendo. Sin embargo, las fronteras entre estos objetivos sociales, políticos, científicos- son obviamente muy borrosas. Sin entrar en el debate acerca de la dificultad de dar cuenta de la "realidad", como investigadoras e investigadores tenemos responsabilidades al momento de hablar de un tema tan relevante en el Chile actual: una responsabilidad ética, de no contribuir a invisibilizar los conflictos socio-ambientales; y una responsabilidad científica, de analizarlos con bases científicas (cruzando información, aceptando los límites de las categorías mismas, objetivando estas categorías de análisis) y de restituir su discusión. Entender de dónde viene 

ambientales en Chile: Reflexiones metodológicas y resultados. Revista de Sociología, 34(1), 81-101. doi: 10.5354/0719-529X.2019.54271

la información y cómo ésta es conceptualizada, categorizada y recopilada -así como las limitaciones de los datos-, es parte de este trabajo de deconstrucción de la investigadora y el investigador (Bourdieu, 2002).

Cuando hablamos de "conflictos socioambientales" tendemos a confundir una categoría de uso, es decir una terminología que todo el mundo emplea y que tiende a utilizarse cada vez más (por medios de comunicación, autoridades y organizaciones sociales) con una categoría de análisis; es decir, una terminología científica. Además, las metodologías que utilizamos tienen una consecuencia sobre la visibilización o no del problema ya que como actores sociales los investigadores contribuyen a definir cuales son los retos sociales y a difundirlos en medios de comunicación, en revistas científicas, en congresos y en intervenciones públicas.

Este artículo presenta una reflexión metodológica sobre los datos disponibles en la actualidad sobre conflictos socio-ambientales en Chile y presenta las primeras reflexiones basadas en el análisis de la base de datos del Observatorio de Conflictos (OdC) del Centro de Estudios de Conflicto y Cohesión Social (COES). Así, se plantea este trabajo como una apertura al diálogo sobre metodologías de investigación cómo definir un tema, cómo recolectar datos e información y cómo articular distintas fuentes de información - para el estudio de los conflictos socio-ambientales en Chile. Este trabajo no pretende ser exhaustivo en mencionar las distintas bases de datos y trabajos que existen sobre el tema. Con sólo mirar la creación de diversos programas académicos, coloquios y agendas de investigación recientes es posible advertir el dinamismo importante que existe al respecto en Chile, y la imposibilidad de nombrarlos todos en estas páginas. Sin duda, todo ello ha colaborado con la construcción de nuestra propia agenda de investigación. Este artículo puede ser útil para las investigadoras y los investigadores, así como también para las organizaciones que buscan acceder a datos claves para observar el panorama de los conflictos socioambientales en Chile. Puede ser útil también para quienes se encuentran construyendo sus objetos de investigación: esperamos que puedan encontrar aquí algunas pistas que les permitan al menos desmitificar el aspecto "práctico" de la investigación.

En esta primera parte se presentarán distintas fuentes de datos que pueden ser de utilidad para los estudiosos de la economía política de los recursos naturales, de los movimientos sociales o de la ecología política. Se dará cuenta de las ventajas de estas bases, siempre y cuando se entiende de dónde vienen los datos, y se abordará algunas preguntas sobre la definición de los términos. En una segunda y tercera parte, se entregarán algunos resultados de una investigación en curso que participa de la definición del "conflicto socio-ambiental", centrándose en uno de sus aspectos: la protesta. De esta forma, se buscará responder a dos preguntas centrales para el mejor entendimiento de las dinámicas de los conflictos socioambientales: ¿quiénes son los ciudadanos que protestan, y cuáles son sus demandas socioambientales? Nos concentraremos sobre estos 

ambientales en Chile: Reflexiones metodológicas y resultados. Revista de Sociología, 34(1), 81-101. doi: 10.5354/0719-529X.2019.54271

dos interrogantes menos estudiadas en trabajos recientes y que nos permiten abordar el rol de los distintos actores sociales, la articulación de sus reivindicaciones y la importancia del aspecto socio-ambiental en las protestas actuales en Chile. El artículo concluye con reflexiones generales para seguir profundizando esta línea investigación.

¿Qué entendemos por conflictos socioambientales?

Existen diferentes iniciativas institucionales y militantes que buscan registrar la conflictividad socio-ambiental en Chile. Se detallan aquí las principales fuentes de datos a nivel nacional: i) el mapa del Instituto Nacional de Derechos Humanos (INDH); ii) los registros del Observatorio de Conflictos Mineros de América Latina (OCMAL) y el Observatorio Latinoamericano de Conflictos Ambientales (OLCA); y iii) el mapa internacional de EJ Atlas. En lo siguiente se destacará la especificidad de cada base de datos, así como los objetivos que buscan sus instituciones y organizaciones.

El mapa de conflictos socio-ambientales del INDH (una institución pública independiente de protección de los derechos humanos, nacida en 2010), es una de las herramientas más utilizadas, tanto por académicos, medios de comunicación como activistas en Chile. Iniciado en 2012 , este mapa tiene como meta visibilizar problemáticas de vulneración de derechos humanos ligados con el medioambiente con el fin de "pone[r] en evidencia la urgencia de que el Estado termine de aprobar normas acordes a los estándares de derechos humanos e implementar la institucionalidad ambiental" (INDH, 2012, p. 250). Basándose en su trabajo de observación de la vulneración de derechos humanos y en la labor de especialistas (consultores externos), el INDH decide levantar información sobre temas socioambientales para construir un primer mapa en 2015, actualizado en 2018 con el aporte de sus distintas sedes regionales. El INDH registra los conflictos desde una perspectiva clara de derechos humanos, a partir de referencias como "el derecho a vivir en un ambiente libre de contaminación" garantizado por la Constitución Chilena y con referencias a estándares internacionales.

En este contexto, los derechos humanos se entienden en relación con otros derechos, como por ejemplo el derecho a la salud, al trabajo, a una vivienda digna, al agua, etc. El INDH ha definido lo que entiende por conflicto en su informe de 2012 (INDH, 2012), como disputas entre varios actores "manifestadas públicamente y que expresan divergencias de opiniones, posiciones, intereses y planteamientos de demandas por la afectación (o potencial afectación) de derechos humanos, derivada del acceso y uso de los recursos naturales, así como por los impactos ambientales de las actividades económicas" (INDH, 2012, p. 246). Para el INDH la manifestación en la esfera pública se entiende como el uso de un repertorio de acción colectiva, tal como una protesta, una acción judicial o una petición, que haya podido tener repercusión en los medios de comunicación. El INDH distingue también varias etapas de los conflictos, entre las que se encuentran conflictos activos (nuevos 
hitos ocurridos durante el último año), latentes (sin nuevos hitos pero sin cierre), cerrados (acuerdo entre las partes o decisión institucional poniendo fin al conflicto) y archivados (estos últimos conflictos ya no aparecen en el mapa, llevan más de un año de cierre y se caracterizan por no haber registrado nuevos hitos). Esta categorización es de utilidad para las investigadoras y los investigadores que buscan identificar procesos de desarrollo de conflictos y factores de cambio, y que intentan comparar formas de evolución. En este sentido, el INDH considera un criterio de persistencia en el tiempo que hay que tener en mente al momento de llevar a cabo investigaciones que buscan una mayor dimensión temporal/histórica. La base de datos que provee el INDH tiene, obviamente, múltiples usos: por ejemplo, permite hacer trabajos comparativos y avanzar o testear hipótesis de trabajo sobre tipos de proyectos, industrias contaminantes, diferencias entre territorios, relaciones entre niveles de desarrollo humano y contaminación, evolución de diferentes conflictos, etc.

Los registros de OLCA (a nivel nacional y de América Latina), de OCMAL (a nivel latinoamericano) y del Environmental Justice (EJ) Atlas (a nivel mundial) siguen una lógica distinta. Son bases de datos abiertas que reposan sobre el trabajo de individuos $\mathrm{u}$ organizaciones sociales que desean registrar una situación conflictiva en términos socio-ambientales.

OLCA es una organización social que busca visibilizar desigualdades estructurales generadas por un modelo económico y social que sus miembros consideran como "depredador"
(OLCA, s.f.). Esta organización tiene varias tareas, entre las que destacan levantar información acerca de situaciones de comunidades afectadas en distintos territorios y acompañar a sus actores en la denuncia de sus problemas y de las causas de éstos. OCMAL tiene una vocación similar; de hecho, OLCA Chile forma parte de OCMAL. Creado en 2007 por varias organizaciones sociales, OCMAL tiene como objetivo defender comunidades locales afectadas por la industria minera y denunciar los efectos de un "mismo modelo" (OCMAL, s.f.) creando redes entre organizaciones de distintos países, generando intercambio de experiencias de luchas y de investigaciones entre ellas y visibilizando sus problemáticas a diferentes niveles (por ejemplo, apoyo a campañas locales y nacionales e incidencia en foros internacionales). Tanto para OCMAL como para OLCA, la tarea de registrar conflictos es una parte de su trabajo, ligado a su rol político de generar redes y acompañamiento a organizaciones sociales. OCMAL mantiene también un observatorio de criminalización de la protesta social por oposición a la minería en América Latina.

La iniciativa del EJAtlas representa una síntesis entre un enfoque militante y/o político -con el objetivo de visibilizar casos y reivindicaciones-y un enfoque científico -entregar material para investigadores que trabajan temas socioambientales. Ligado a la Universidad Autónoma de Barcelona, dirigido por Leah Temper y Joan Martínez Alier y coordinado por Daniela Del Bene (Temper, Del Bene, \& Martínez, 2015), EJAtlas entrega un panorama de conflictos socio-ambientales a nivel mundial, los cuales se 

ambientales en Chile: Reflexiones metodológicas y resultados. Revista de Sociología, 34(1), 81-101. doi: 10.5354/0719-529X.2019.54271

caracterizan por remitir a poblaciones que se encuentran luchando por la justicia ambiental. Esta iniciativa define el conflicto como las "movilizaciones de comunidades locales contra actividades económicas especificas donde los impactos ambientales son elementos claves de sus agravios" (Temper, Del Bene \& Martínez, 2015, pp. 261-262, traducción propia). La metodología está basada en la investigación colaborativa y en una perspectiva de activist-led research (Temper, Del Bene, \& Martínez, 2015, p. 258), considerando que las poblaciones afectadas no sólo son objeto de estudio, sino actoras de la producción de conocimiento.

En términos específicos, la base se construye a través de dos pasos. Primero, las informaciones son levantadas por activistas denominados frontline comunities and collaborators, que colaboran directamente con los investigadores (OCMAL es uno de estos activistas). Después, los equipos de EJAtlas se encargan de seleccionar la información, moderar, validar y publicar en la página del Atlas según 10 categorías de conflicto (la cuales no están vinculadas únicamente a actividades de extracción propiamente tal). Los tres principales criterios de registro son i) la presencia de una actividad económica o una legislación con impactos medioambientales y sociales negativos, ii) la existencia de reivindicaciones y movilizaciones por parte de organizaciones de justicia ambiental que apuntan al resultado o probable resultado de esta actividad, iii) todo caso registrado debe tener respaldo en al menos un medio de comunicación. Además de cartografiar los conflictos, el Atlas permite acceder a información sobre las organizaciones movilizadas, la presencia de organizaciones indígenas, la capacidad de las acciones colectivas para detener los proyectos contenciosos o el efecto de un cambio de legislación. En este sentido, el Atlas permite trabajar los efectos que puede tener la movilización sobre el desenlace de los conflictos socio-ambientales.

Un excelente ejemplo de cómo usar esta variedad de información para plantear un objeto de investigación sobre conflictos socio-ambientales es la iniciativa llevada por el proyecto Fondecyt "Lo que los conflictos producen", mencionado anteriormente. Los investigadores utilizaron la base de datos del INDH junto con complementos de OLCA para poder analizar una diversidad de conflictos alrededor del territorio, y eligieron concentrarse sobre conflictos no-urbanos (Delamaza et al., 2017). A partir de estas informaciones reconstruyeron su propia base de datos, complementando los casos con información de prensa, del Sistema de Evaluación Ambiental (SEA) y con fuentes secundarias, particularmente las analizadas por organizaciones territoriales y ONGs. Estos investigadores escogieron como fecha de inicio el año 2005 para avanzar en la comprensión del proceso de politización de los conflictos socioambientales, fecha que corresponde a la movilización en Valdivia contra una planta de celulosa por la protección de los cisnes de cuello negro (Sepúlveda \& Villarroel, 2012). Después, clasificaron los conflictos en distintos sectores para tratar de abarcar la especificidad sectorial, lo que les permite demostrar que los sectores de energía y minería son aquellos donde se 

ambientales en Chile: Reflexiones metodológicas y resultados. Revista de Sociología, 34(1), 81-101. doi: 10.5354/0719-529X.2019.54271

encuentran más conflictos. De esta forma, la base de datos permite desarrollar futuros trabajos sobre los sectores conflictivos (Maillet \& Albala, 2018).

El reto de registrar y de categorizar

Hemos mostrado que existen diferentes instituciones y organizaciones involucradas en la construcción de conocimiento sobre conflictos socio-ambientales; por consiguiente, hay varios actores contribuyendo a construir un problema público acerca del tema (Neveu, 2015). Esquemáticamente, tendemos a tener dos lógicas, a veces complementarias. La primera, basada sobre trabajos prioritariamente científicos, buscan registrar para poder generar datos, entregar un panorama de la situación y trabajar hipótesis. La segunda, basada sobre trabajos que se autodenominan políticos o militantes, buscan dar visibilidad a varias situaciones y procesos para incidir en la agenda política, presionar sobre políticas específicas y conectar comunidades que estén viviendo situaciones parecidas. Como investigadoras e investigadores debemos tener en cuenta estos distintos enfoques al momento de hacer uso de los datos. Con esto no se quiere decir que existen mejores formas de trabajar, sino que estos trabajos apelan a distintos registros de legitimidad (legitimidad científica, política, social) y por consecuencia debemos tomar en cuenta estas para usar los datos en las investigaciones. Se señalan además otras dificultades en el registro de los conflictos: la primera tiene que ver con la dimensión temporal del registro y con la asociación común entre conflicto y proyecto; y la segunda, con lo que entendemos por "conflicto" y por "socioambiental".

Primero, un elemento clave es la dimensión temporal: se debe prestar atención al periodo que se considera estudiar, y tener cuidado al momento de analizar un fenómeno "con lupa". Cabe notar que a partir de 2010, la creación de los Tribunales Ambientales (TA) y de la nueva normativa ambiental ha influido en la dinámica de los conflictos socio-ambientales, así como en la apertura de una nueva agenda de investigación. Las principales bases de datos expuestas en este artículo son bastante recientes, y pocas consideran años anteriores a los 2000. Es necesario tener en cuenta este sesgo de temporalidad; en ciertos casos los conflictos "recientes" pueden haber existido antes de los 2000, o tener raíces en otras movilizaciones. A pesar de una creciente visibilidad por los conflictos relacionados con proyectos energéticos y mineros, cabe señalar que no todos los conflictos están necesariamente ligados a proyectos contenciosos que se han ingresado al SEA. Por ejemplo, existen conflictos que dan cuenta de situaciones de contaminación histórica, donde los proyectos en cuestión fueron aprobados antes que existiera el SEA y los TA en Chile. Ejemplos de estos casos son los relaves mineros abandonados registrados por Fundación Relaves (s.f.), que están siendo visibilizados como problemas socio-ambientales por medio de la acción de organizaciones sociales. Existen otros conflictos socio-ambientales que no están relacionados con un proyecto específico, sino con 
varias actividades contaminantes, como es el caso de "zonas de sacrificio". Un caso relevante, aun cuando bastante invisibilizado, es la situación de la comuna de Til Til en la Región Metropolitana, donde existen numerosas actividades "contaminantes" entre las cuales se pueden registrar relaves mineros, actividad industrial y de minería, actividad agroindustrial, actividad de tratamiento de residuos orgánicos e industriales (INDH, 2018).

Segundo, las delimitaciones y definiciones del conflicto pueden variar. La definición de un conflicto implica un acercamiento a las causas objetivas y subjetivas del conflicto, y supone tomar una decisión conceptual. La alianza de lo social con lo ambiental permite ligar dos retos importantes de la vulneración, tanto de derechos humanos como del medioambiente. Así, la definición de "socio-ambiental" reafirma la relación estrecha entre vulneración de las personas y de su medioambiente, a través de la afectación a su salud por la contaminación del aire, suelo, agua, etc. Ejemplo de esto fue el caso por contaminación por el plomo en Arica estudiado por Arriagada (2012). La utilización del término "territorio" como sinónimo de "medioambiental", en algunas ocasiones, merece dos aclaraciones. Por una parte, los estudios que hablan de territorio consideran la relación que las personas tienen con su entorno (tierra, recursos naturales, lugar específico) y abordan la problemática del sentido de pertenencia (Beuf, 201; Di Méo, 1998). Por otra, el uso de la terminología "territorio" tiende a convocar mayormente a las geógrafas y los geógrafos, quienes han estado más atentos en sus investigaciones a las nociones de espacio vivido y apropiado. Trabajos recientes insisten en la necesidad de adoptar un enfoque territorial para seguir con el estudio de los conflictos socioambientales (Aliste \& Stamm, 2014). De esta forma, el ejercicio mismo de definición de los conflictos como "territoriales" y/o "socioambientales" nos lleva a pensar las reivindicaciones, a partir de la relación que tienen las y los habitantes con su entorno y las percepciones sobre éste. En definitiva, se trata de estudiar las maneras de politizar, entendiendo la politización como un doble fenómeno: por un lado, el ejercicio de la generalización a partir de una situación (lo que me pasa a mí aquí también está ocurriendo a otras personas en otros lugares); y por el otro, el ejercicio de denominación sobre una situación como problemática política (lo que me está pasando interroga la distribución del poder, de los recursos y las desigualdades históricas y actuales, y me lleva a pensar los modelos de desarrollo y de democracia).

\section{MÉTODO}

En esta segunda parte quisiéramos dar cuenta de una investigación en curso y que tiene como punto de partida el análisis de la base de datos del OdC/COES (COES, 2018). Esta base permite 

ambientales en Chile: Reflexiones metodológicas y resultados. Revista de Sociología, 34(1), 81-101. doi: 10.5354/0719-529X.2019.54271

otra mirada sobre la conflictividad socioambiental con foco en los eventos de protesta para, desde esta perspectiva, profundizar en la comprensión de los procesos de politización de los problemas socio-ambientales en Chile y observar nuevas formas de movilización en la sociedad chilena, que ocurren con la emergencia de nuevos actores.

Aportes y límites de la base de datos del OdC/COES

En 2015 el COES decide generar una base de datos para analizar la conflictividad en el país. Así, el OdC nace siguiendo la experiencia ya desarrollada por el Observatorio de Huelgas, y busca registrar las protestas que ocurren en el país de manera sistemática, organizando tipos de protesta según una variedad de criterios tales como tipos de demandas y repertorios de acción utilizados. Se considera una variedad de 18 medios de comunicación (prensa escrita y digital y radios; medios nacionales y regionales). El método es el del Protest Event Analysis, y se entiende "protesta" como "una manifestación colectiva en el espacio público, que ocurre en un momento y lugar específicos (bloqueo de autopistas, manifestaciones, marchas, tomas de edificios o espacios públicos, barricadas, huelgas y otros)" (COES, 2017, p. 5). La base de datos permite registrar protestas que ocurren en un lugar y tiempo específicos, así como tener en cuenta episodios de protestas (varias protestas con el mismo motivo en diferentes lugares en el mismo día) y procesos contenciosos (episodios que ocurren en jornadas sucesivas). El OdC se inspira de las categorías de campos de conflictividad de la base del Programa de Naciones Unidas para el Desarrollo (PNUD) (Calderón, 2012) que contempla 3 categorías (conflictos por reproducción social, conflictos institucionales y conflictos culturales) a la cual fue añadida una cuarta categoría sobre conflictos territoriales por el COES. Además la base de datos del OdC incluye más medios de prensa en el análisis. Se trata sin duda de un avance para la comprensión de la conflictividad en Chile, pues constituye la base de datos más importante y completa sobre protestas en el país. Al mismo tiempo, permite tener una visión macro del dinamismo de la ciudadanía, así como del malestar que existe en varios sectores al respecto. La existencia de una base como ésta permite también abrir una agenda de investigación capaz de entregar reflexiones sobre el estado de la democracia en el país, sobre los sentidos de la ciudadanía y la relación entre la sociedad y el Estado, por mencionar algunos tópicos.

Sin embargo, como toda base de datos, es necesario considerar también sus limitaciones. El primer límite está ligado a la metodología de recolección de información; esto, dado que el Protest Event Analysis levanta información sólo de lo que aparece en la prensa. Olivier Fillieule, quien ha estudiado la evolución de la preocupación periodística por los problemas ambientales en Francia, demuestra que el análisis a partir de la prensa implica tres tipos de sesgos: accuracy (exactitud), selectivity (selectividad de las noticias) y systematicity (sistematicidad de los sesgos) (Fillieule, 2007). 
Así, se puede señalar que este primer límite está relacionado con la producción de resultados basados en información de segunda mano, y con la necesaria capacidad crítica que se debe tener respecto de estas informaciones. El segundo lleva a tomar en cuenta el trabajo mismo de los periodistas y de las empresas de prensa, vale decir: i) la forma en que seleccionan las noticias pertinentes; ii) su capacidad para tener o no periodistas en distintas partes del país; iii) su capacidad para reportar un evento que convoca a pocas personas; y iv) su capacidad para reportar un evento violento/no violento, rutinario o nuevo, etc. En este sentido, la selectividad no sólo depende de los sesgos clásicos de la prensa y de sus empresas, sino que depende también de los eventos mismos, la naturaleza de la protesta, los modos de organización de los manifestantes y los destinatarios. Tercero, la sistematicidad de los sesgos depende de los ciclos de atención mediática y de la concurrencia entre noticias: una protesta puede tener más o menos atención mediática según haya o no un evento "mayor" que reportar al mismo tiempo.

Para el contexto chileno, la base de datos del OdC tiene otros límites que tenemos que tomar en cuenta. Dada la organización nacional de los medios de prensa, los conflictos urbanos de la Región Metropolitana (RM) podrían aparecer con menos frecuencia en la prensa. En efecto, dado que ésta cubre mayoritariamente eventos nacionales, podría haber una menor atención hacia lo local. Para el estudio de los conflictos en la RM, Aliste y Stamm (2016) proponen incluir datos de otras fuentes, como los tribunales de justicia (TA y Corte Suprema) y redes sociales (por ejemplo, grupos de Facebook). Además, algunas protestas podrían ser invisibilizadas por la importancia de las estrategias comunicacionales de las empresas, por los intereses económicos que pueda haber acerca de ciertos proyectos, cómo lo señalan Maillet, Delamaza y Martínez (2017) y también por la poca pluralidad de la información escrita (pocos grupos periodísticos).

Teniendo en cuenta estas ventajas y limitaciones, voy a explicar con mayor detalle cómo se seleccionó la muestra para entender las protestas socio-ambientales.

¿Qué son las protestas socio-ambientales? Selección de la muestra

Es necesario dar cuenta del ejercicio de definición, categorización y re-codificación de los datos para analizar lo que entenderemos por protestas socio-ambientales. Antes de seleccionar la muestra se construyó una definición de trabajo, es decir, una primera definición que permite avanzar y cuestionar a la vez el objeto "socio-ambiental" y la conflictividad. Definimos la protesta con componente socioambiental como una acción colectiva que cuestiona el acceso a un territorio o sus recursos naturales (suelo y subsuelo); a la concepción cognitiva, cultural, colectiva o identitaria de un territorio y de la forma de vivir del territorio, en el territorio y con el territorio; y cualquier afectación o cambio que se genere o que se requiere según los actores sociales.

La base de datos del OdC tiene múltiples usos y escogimos entrar por la variable "elemento de 

ambientales en Chile: Reflexiones metodológicas y resultados. Revista de Sociología, 34(1), 81-101. doi: 10.5354/0719-529X.2019.54271

demanda" la cual identifica las demandas declaradas por los participantes de la protesta o declaradas en una convocatoria (representa 143 códigos diferentes en total) y por la variable "campo de conflictividad" (4 códigos principales diferentes).

En una primera etapa se revisaron los elementos de demanda que correspondían a nuestra definición antes mencionada. Tres grupos de demandas corresponden a lo que identificamos como socio-ambientales y territoriales, las cuales son: i) ecologistas, ambientalista; ii) regionalistas, urbanas y/o locales; y iii) pueblos originarios.

Tabla 1. Protestas relacionadas con temas socioambientales 2012-2017.

\begin{tabular}{lcc}
\hline Demanda & Frecuencia & Porcentaje \\
\hline $\begin{array}{l}\text { Pueblos } \\
\text { originarios }\end{array}$ & 573 & 34.0 \\
Ecologistas & 148 & 8.8 \\
Regionalistas & 965 & 57.2 \\
\hline Total & 1686 & 100.0
\end{tabular}

Nota: elaboración propia en base a datos del observatorio de conflictos COES.

En una segunda etapa decidimos establecer una nueva base de datos recodificando los elementos que son de interés para nuestro estudio. Por la complejidad de los campos de conflictividad asociados a grupos originarios en la base de datos se decidió en adelante considerar solamente los dos primeros grupos mencionados -los movilizados por demandas ecologistas (6 tipos de demandas codificados en la base) y por demandas regionales/locales (también estas representan 6 tipos de demandas codificados en la OdC). En efecto, a pesar de compartir características comunes con numerosos grupos ambientalistas y locales, existen pocas acciones colectivas entre grupos originarios y otros actores. Las protestas interétnicas, es decir dónde confluyen actores indígenas y no indígenas representan menos del $10 \%$ de las acciones contenciosas registradas en la base de datos. Esto se puede explicar por las experiencias previas de luchas conjuntas entre grupos indígenas, particularmente mapuche, y organizaciones ambientales, las cuales han dejado divisiones en los petitorios y en las formas de movilización, como fue el caso en la oposición al proyecto Ralco (Carruthers \& Rodríguez, 2009; Martínez \& Delamaza, 2018). Además, análisis previos de las protestas mapuches basadas en el OdC (Romero, Castro, Jaramillo \& Alister, 2017) demuestran que para el periodo 2015-2016 el campo de conflictividad "tierra y territorio" representa el 30.3\% del total de las acciones contenciosas relacionadas con el pueblo mapuche, y el $45.5 \%$ se relaciona con "identidad, memoria y patrimonio", lo cual hace difícil el ejercicio de identificación de las demandas socioambientales con esta metodología. Esta decisión metodológica no significa que las protestas de pueblos originarios no incluyan demandas socioambientales en sus reivindicaciones (la terminología misma de "tierra y territorio" así como numerosos estudios sobre el tema lo han demostrado) ni tampoco que las demandas ecologistas y regionales/locales no incluyan una 
articulación con demandas de los pueblos

originarios.

\section{RESULTADOS}

Resultados del estudio de las protestas socioambientales 2012-2017

Entre 2012 y 2017 se produjeron en Chile 1,113 eventos de protestas socio-ambientales. Se trata de un número importante, si consideramos que representa un $10.8 \%$ del total de protestas ocurridas en Chile durante este periodo, ubicándose en el tercer lugar luego de las protestas laborales $(42.2 \%)$ y las relacionadas con educación (16.2\%). El 2012 es el año más conflictivo para los retos socio-ambientales, con 281 eventos; en los siguientes tres años el número de protestas disminuye, repuntando nuevamente en 2016 con 193 eventos. Estas protestas se manifiestan a través de repertorios pacíficos, como marchas o manifestaciones, y por acciones "disruptivas no violentas", como cortes, barricadas o tomas de rutas. Siguiendo la definición de trabajo mencionada anteriormente, se consideran varios tipos de demandas, y se propone abordar algunos de los procesos de politización para comprender quiénes son los actores movilizados, para avanzar así en el estudio acerca de a quiénes apuntan sus demandas.

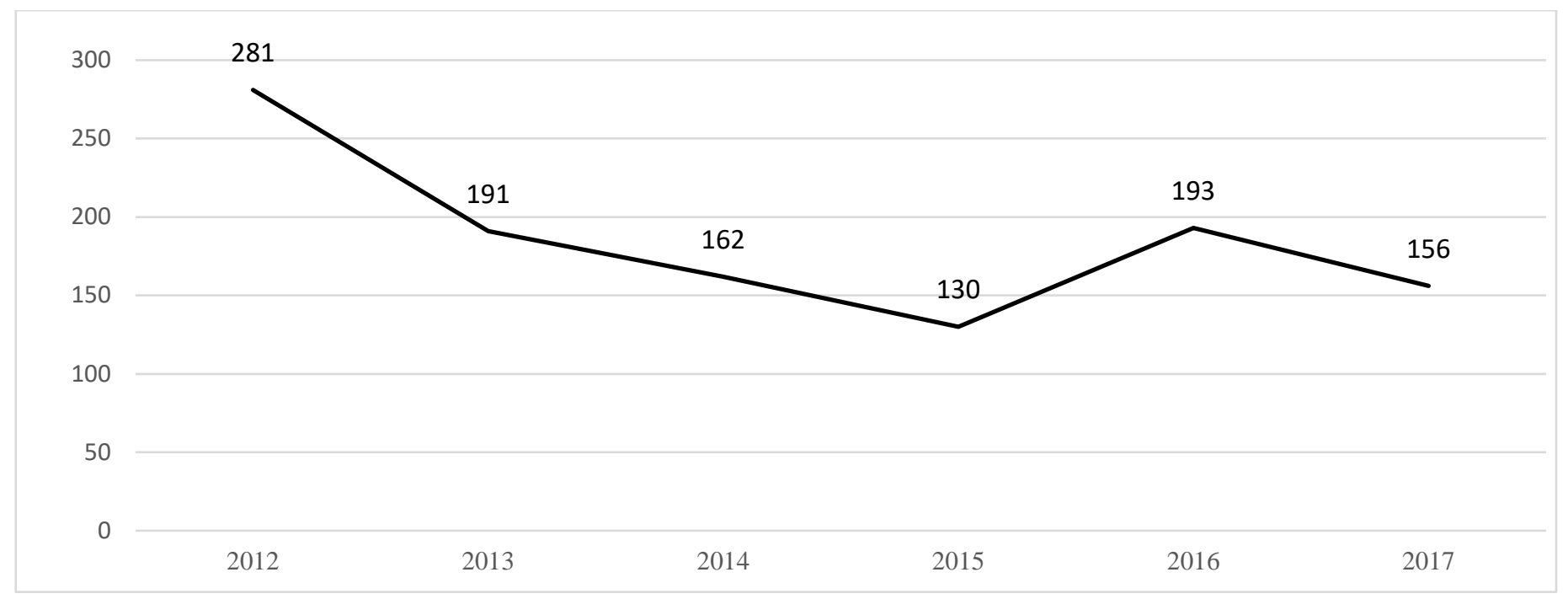

Figura 1. Número de protestas socio-ambientales por año. La figura fue elaborada en base a datos del observatorio de conflictos COES. 

ambientales en Chile: Reflexiones metodológicas y resultados. Revista de Sociología, 34(1), 81-101. doi: 10.5354/0719-529X.2019.54271

Demandas: imbricación de motivos y distribución de las protestas en todo el territorio chileno

Dentro de lo que consideramos protestas socioambientales, existe una variedad de tipos de demandas diferentes, siendo las más relevantes en términos cuantitativos las relativas al desarrollo local (17.6\% del total), seguidas por conflictos por la construcción de infraestructura urbana (17\%), contaminación en la zona (14.6\%) y proyectos energéticos en la zona (14.2\%).

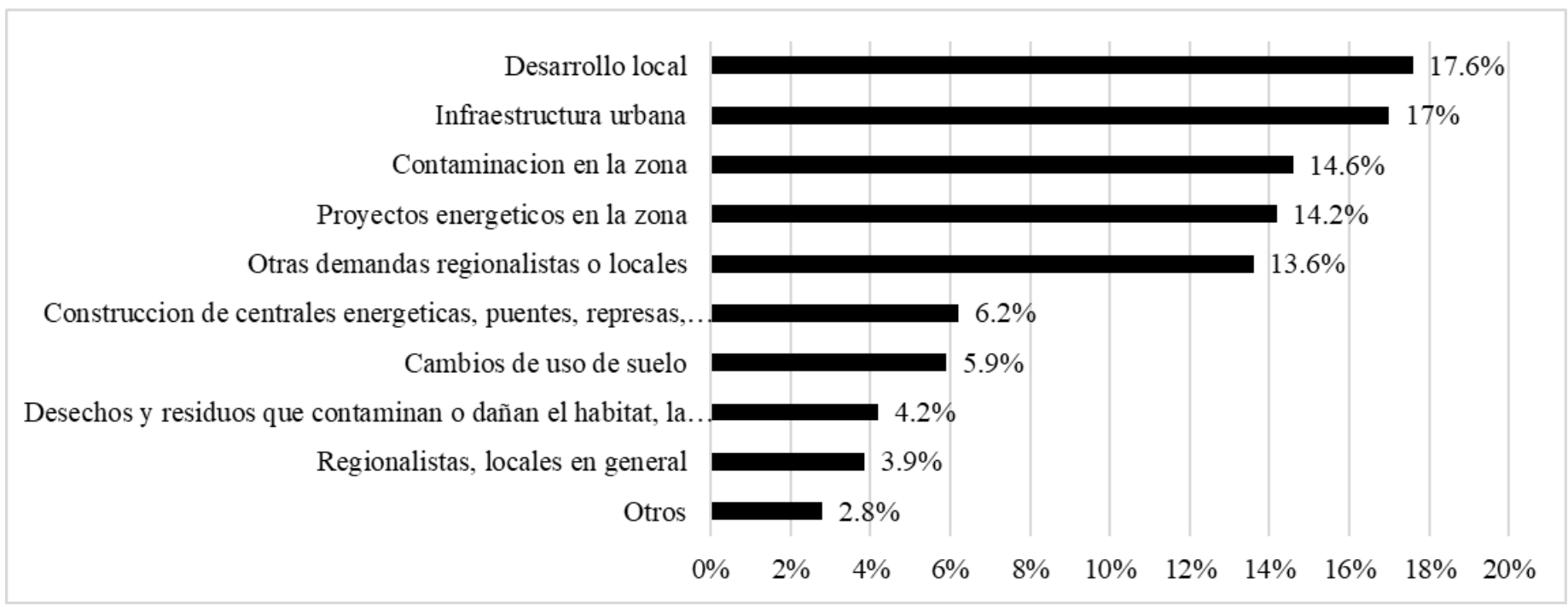

Figura 2. Tipos de demandas en protestas socio-ambientales. La figura fue elaborada en base a datos del observatorio de conflictos COES.

Si se aíslan las protestas por temas de energía, por el hecho que éstas se den por oposición a la implementación local de proyectos energéticos (categorizados como demandas regionales, urbanas y/o locales) o por una causa ecologista más general (categorizados como demandas ecologistas, ambientales), se alcanza 227 protestas, lo que corresponde a un $20.4 \%$ del total de protestas socio-ambientales. Es posible observar que las protestas con componente socioambiental se dan mayoritariamente en territorios urbanos (78.1\%), mientras que sólo el $21.9 \%$ se materializa en territorios rurales. Eso significa que la mayoría de los actores sale a protestar en las ciudades, donde logran ser visibles, pero no necesariamente significa que su territorio de origen -o el lugar afectado por un problema específico- sea urbano.

$\mathrm{Al}$ distribuir las protestas por región, se obtiene que ellas ocurren en todo Chile, y que el 89.3\% ocurre fuera de la RM. Las protestas se concentran mayoritariamente en las regiones de Aysén, Metropolitana, Araucanía, Valparaíso y Los Lagos. Relacionando estos resultados con el número de habitantes por región (INE, 2018), las regiones más contenciosas son aquellas más aisladas del centro, como Aysén, Atacama, Los Ríos, Arica y Parinacota y Antofagasta. 

ambientales en Chile: Reflexiones metodológicas y resultados. Revista de Sociología, 34(1), 81-101. doi: 10.5354/0719-529X.2019.54271

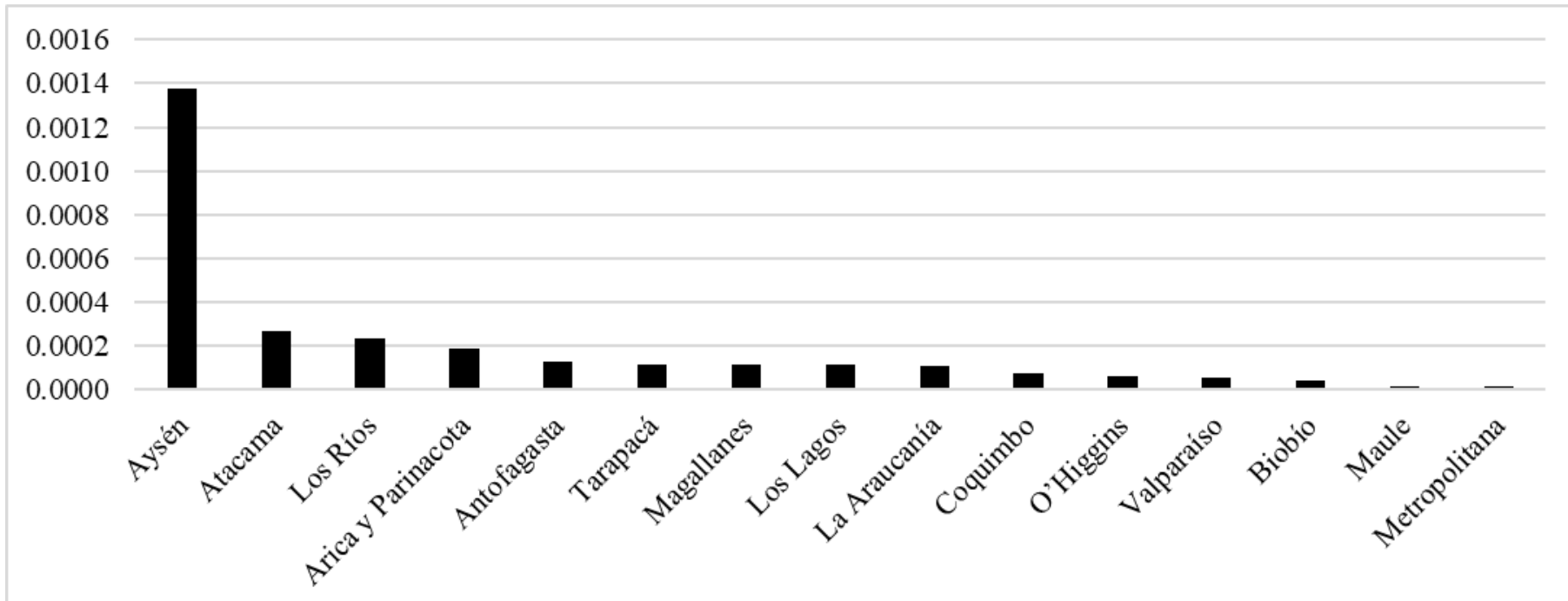

Figura 3. Número de protestas por habitante según región. La figura fue elaborada en base a datos del observatorio de conflictos COES.

Finalmente, al tomar las 6 regiones más contenciosas y observar la distribución de protestas por año, como se observa en la figura 4, se obtiene que en general la distribución de conflictos es homogénea a excepción de Aysén, que concentra sus protestas en 2012 (año en el cual se materializa el Movimiento Social por Aysén).

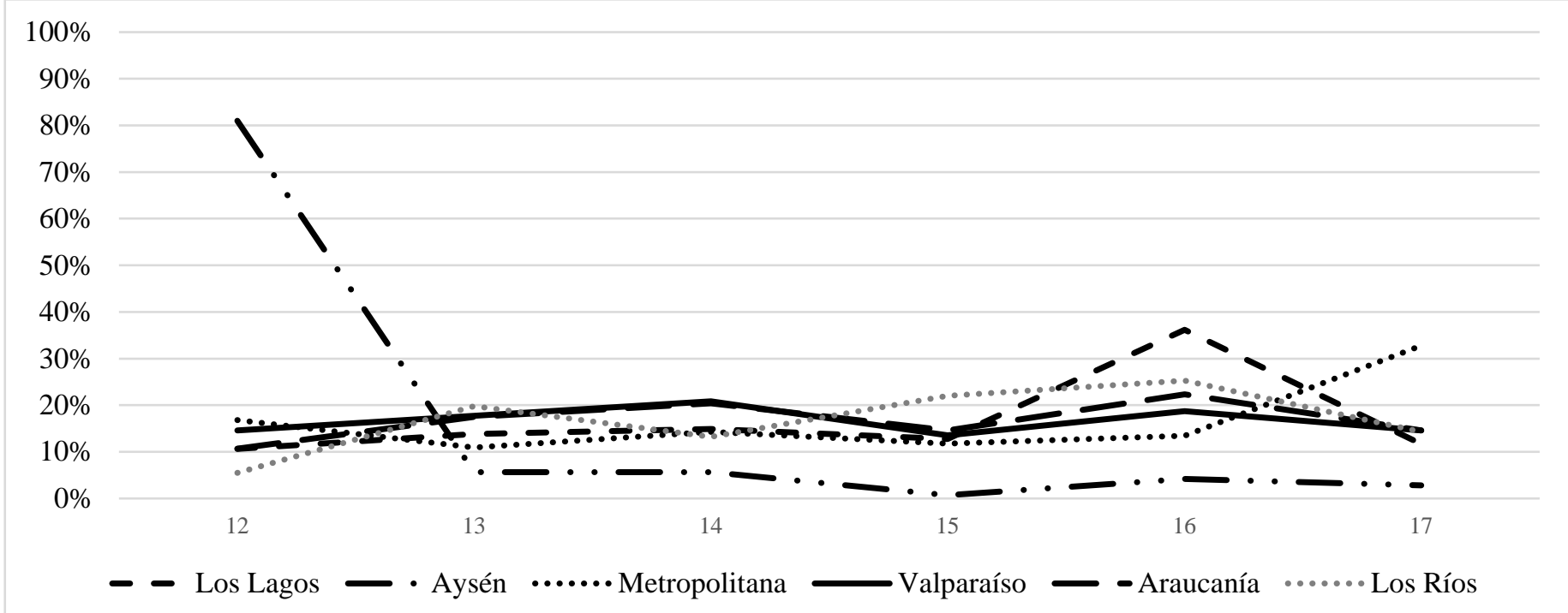

Figura 4. Distribución de protestas por año según región. La figura fue elaborada en base a datos del observatorio de conflictos COES. 

ambientales en Chile: Reflexiones metodológicas y resultados. Revista de Sociología, 34(1), 81-101. doi: 10.5354/0719-529X.2019.54271

Actores: los ecologistas no son los principales actores de las protestas socio-ambientales a lo largo del territorio chileno

Otra observación que se puede hacer a partir del estudio de la base de datos del OdC es la siguiente: los primeros afectados son los actores principales de las protestas socio-ambientales en Chile. La mayoría de los actores que llevan acciones colectivas no son las grandes organizaciones ambientalistas o ecologistas pertenecientes a las diferentes corrientes de defensa del medioambiente en Chile (Carruthers, 2001), lo que parece confirmar que en Chile no existe un movimiento socioambiental homogéneo (Schaeffer, 2016).

El 69.9\% de los grupos sociales identificados son "residentes o vecinos", ciudadanos organizados a un nivel más pequeño de la escala administrativa, que no forman parte de organizaciones formales dedicadas exclusivamente al medioambiente. Así, se puede decir que se trata de protestas lideradas por los primeros afectados, organizados en coordinadoras locales, juntas de vecinos o comités de ciudadanos alrededor de un tema específico. Son los lugareños, vecinos, residentes de un lugar o habitantes de un territorio quienes se movilizan en contra de proyectos o situaciones que consideran afectan su medioambiente, salud, forma de vivir o de relacionarse con su hábitat, o actividad social y económica. La presencia de este actor "ciudadano" nos lleva a reflexionar sobre la territorialidad de estas protestas y la fuerte pertenencia de los ciudadanos con su espacio: el barrio, el pueblo, el cerro, la localidad (sobre esta relación, y la construcción de distintas relaciones con el territorio, ver Biskupovic \& Stamm, 2016). La poca afiliación de los actores de las protestas con organizaciones más formales y profesionales da cuenta de un proceso distinto de politización y de otras formas de llevar a cabo acciones colectivas. El medioambiente aparece como una ventana de expresión de un sufrimiento multidimensional, ligado a situaciones más generales de vulnerabilidad social y económica, particularmente en regiones lejanas de los centros del poder.

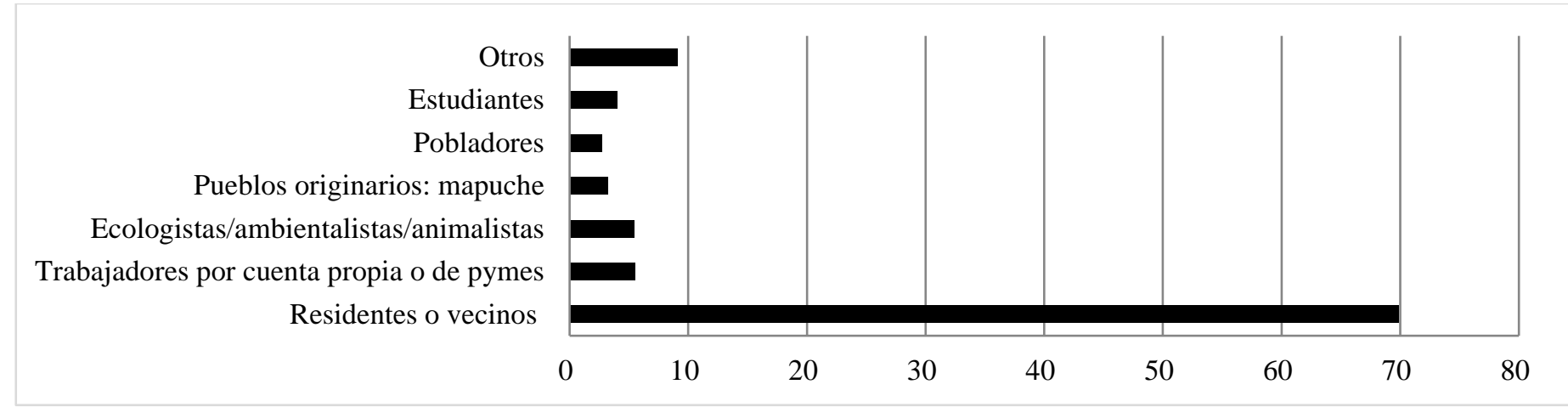

Figura 5. Grupos sociales en protestas socio-ambientales. La figura fue elaborada en base a datos del observatorio de conflictos COES. 

ambientales en Chile: Reflexiones metodológicas y resultados. Revista de Sociología, 34(1), 81-101. doi: 10.5354/0719-529X.2019.54271

Si bien el medioambiente es uno de los componentes de las demandas, éstas se articulan con condiciones de vida en general como contaminación, problema de desechos que dañan el hábitat y/o la salud, y condiciones de trabajo más generales afectadas por una actividad específica como lo resalta la presencia del grupo social "trabajadores por cuenta propia o de pymes". Múltiples protestas apuntan a que lo que se queda en el territorio son sólo los efectos adversos de las inversiones, como la contaminación y sus respectivos impactos sobre el medioambiente y la salud de la población. De esto da cuenta la tercera demanda más amplia de las protestas, por contaminación en la zona en la figura $2(14.6 \%)$.
Destinatarios y politización: el Estado como objetivo de las protestas

El objetivo de los actores sociales movilizados entrega información valiosa sobre los procesos de politización, ya que dirigen sus demandas al actor que consideran más legítimo para intervenir, o más eficaz para solucionar sus problemas. Así, el objetivo de la protesta da cuenta de un ejercicio de otorgamiento de responsabilidad y de una reflexión sobre el actor garante de preservar derechos y de solucionar problemas. Como muestra la figura 6, el principal objetivo de las demandas son gobierno o autoridades regionales o locales, notoriamente por sobre gobierno o autoridades nacionales o empresas privadas -a excepción del año 2012, donde las protestas que apuntaban al gobierno central alcanzaron casi el 70\%.

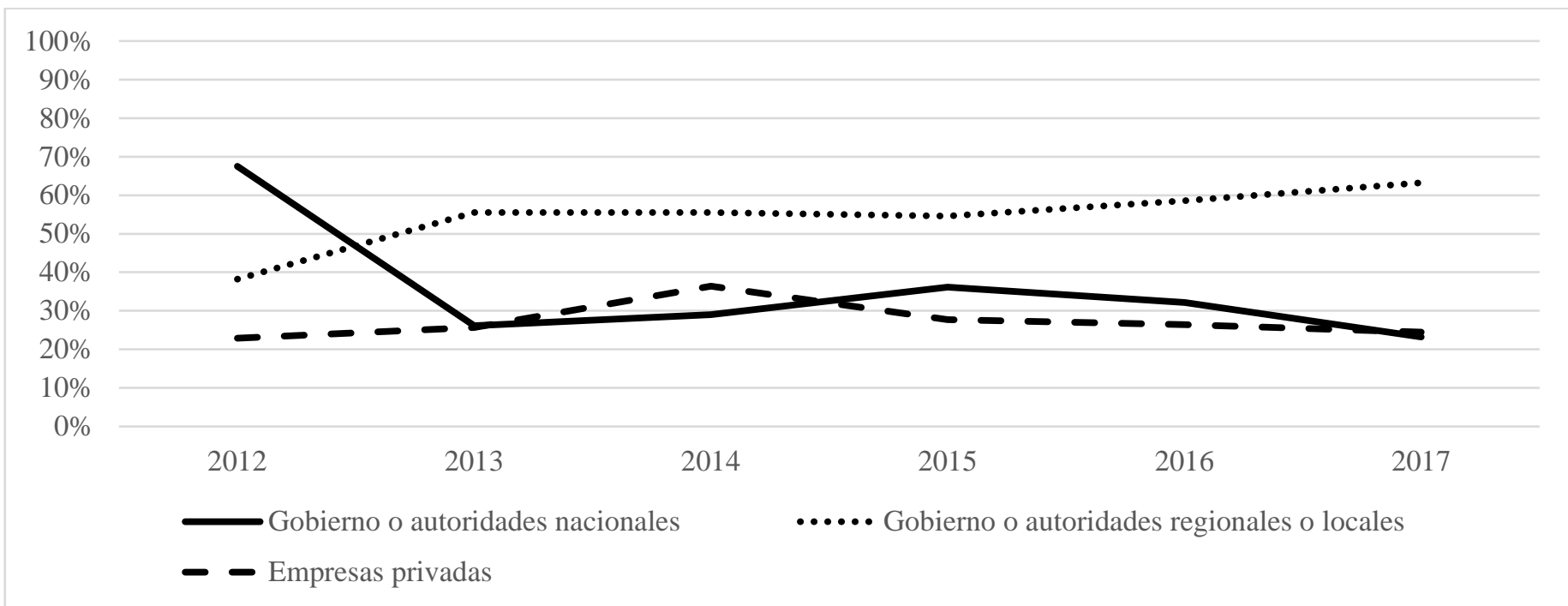

Figura 6. Target de protestas socio-ambientales. La figura fue elaborada en base a datos del observatorio de conflictos COES. 

ambientales en Chile: Reflexiones metodológicas y resultados. Revista de Sociología, 34(1), 81-101. doi: 10.5354/0719-529X.2019.54271

Podemos identificar ahí dos lógicas de movilización y procesos de politización. Por un lado, y siguiendo a Valenzuela, Penaglia y Basaure (2016), el actual ciclo de protestas territoriales no puede ser reducido a un "mero localismo defensivo ante un daño ambiental" (p. 226), sino que ha evolucionado gradualmente hacia una construcción de discursos que apuntan a demandas como una mayor autonomía política y una distribución de riquezas más equitativa para los territorios. Reflejo de ello son las demandas concretas a las que se adscriben las protestas que se clasifica en esta investigación como "socio-ambientales". En efecto, las principales demandes socio-ambientales son por desarrollo local, que abarca aspectos como mejor legislación, más fondos y condiciones generales para regiones o localidades e inquietudes frente a la construcción de infraestructura urbana, que refleja la falta de inversión pública en regiones. Ambos elementos de demanda concentran el $34.6 \%$ de las protestas, y en ellas se justifica que su objetivo sean autoridades gubernamentales.

Por otro lado, en tercer y cuarto lugar se encuentran las protestas por contaminación y por proyectos energéticos en la zona, las cuales se vinculan generalmente a proyectos de inversión llevados a cabo por empresas. Resulta interesante que las demandas no apunten a actores privados y sí a actores públicos, particularmente en un contexto de un neoliberalismo fuerte, donde el Estado se reduce a sus funciones mínimas y el mercado parece ser el ente regulador de todos los aspectos de la vida social (Garretón, 2012). El objetivo predominante resulta aún más paradójico en un periodo de creciente desconfianza en las instituciones y desaprobación a los gobiernos (Joignant, Morales \& Fuentes, 2016). A pesar de ello, parece que la ciudadanía sigue apelando a algunas de las instituciones tradicionales, y a sus funciones de garantes de derechos.

Durante la última década se ha producido una expansión de la Responsabilidad Social Empresarial como estrategia de negocios (Tironi \& Zenteno, 2012). Esto ha significado en la práctica que las empresas, a través de diferentes programas y proyectos sociales, sustituyan la acción del Estado en aquellos territorios que se han visto "abandonados" por éste. Resultaría lógico, en este contexto, que la ciudadanía tendiese a buscar solución a sus problemas locales en este actor, que generalmente cuenta con más recursos que las autoridades locales. Sin embargo, apuntar a la empresa significa buscar soluciones específicas para problemas específicos, como compensaciones a cambio de las externalidades de las inversiones. Apuntar a actores públicos implica acceder a otro tipo de soluciones que superan el ámbito de lo local, como mayor regulación a los proyectos de inversión, protección institucional frente a los efectos adversos, cambios en los instrumentos de planificación que permiten las inversiones, mayor participación en las decisiones que afectan al territorio, etc. Estas soluciones son a su vez replicables en otros territorios, e implican cambios en la forma en que se distribuye el poder dentro de éstos. Por todos estos motivos se sugiere un proceso de politización. Finalmente, sería interesante abordar la preferencia de los actores de la protesta por apuntar a las 
autoridades regionales o locales en desmedro de las autoridades nacionales. Proponemos que esto podría ser un ejemplo del creciente interés por la descentralización que ha surgido desde diferentes regiones de Chile.

Como vemos, la base de datos del OdC/COES ofrece múltiples usos y entrega resultados útiles para seguir indagando sobre la conflictividad socio-ambiental en Chile. Permite abrir una agenda de investigación, generar hipótesis inductivas y/o testear hipótesis deductivas sobre actores de la protesta, principales demandas, lugares de las protestas, destinatarios. En nuestro caso, permite generar algunas reflexiones para seguir con la investigación cualitativa, para tratar de entender cuáles son las otras estrategias (aparte de la protesta) desarrolladas por los habitantes que buscan soluciones a sus problemáticas y para entender, a partir de un estudio de caso, las distintas percepciones que tienen los actores sobre las injusticias ambientales y la articulación de lo social con lo territorial.

\section{DISCUSIÓN Y CONCLUSIONES}

Este artículo tuvo dos objetivos. El primero fue exponer una reflexión metodológica sobre el uso de diferentes bases de datos que existen hoy para estudiar los conflictos socio-ambientales. Aquí, se buscó demostrar la importancia de comprender de dónde vienen los datos, sus metodologías y los objetivos de sus iniciadores, así como también describir la necesidad de interrogarse sobre las definiciones que utilizamos de manera común. Definir una situación como un "conflicto socio-ambiental" o "conflicto socio-territorial" tiene implicancias metodológicas, pero también sociales y políticas, porque contribuimos a dar visibilidad -o bien, a invisibilizar- situaciones vividas por la población. El segundo objetivo fue dar cuenta de nuestro propio proceso de investigación sobre la base de datos del OdC/COES, proponer una forma de uso y recodificación y avanzar algunos resultados preliminares para despertar hipótesis de trabajo. En este trabajo, se propuso dichas hipótesis en forma de reflexiones.

Ahora bien, a modo de conclusión quisiéramos mencionar algunos elementos claves para seguir con el estudio de los conflictos socio-ambientales de manera más cualitativa.

Con este acercamiento a la caracterización de la protesta socio-ambiental, invitamos a seguir explorando los procesos de politización de las demandas categorizadas como "ecologistas" y "locales". Para ello proponemos profundizar la investigación en torno a los dos actores propuestos en este artículo: por un lado, el demandante "residente o vecino", entendido como actor emergente y mayoritario de la protesta, y por otro, el demandado, como el actor escogido por los manifestantes para ser foco y canalizador de sus demandas. Lo anterior, en torno a las dos lógicas de movilización identificadas: vale decir, las demandas por desarrollo local y las demandas por los efectos de 
la contaminación. De esta manera, si bien no se puede hablar de una dinámica de movilización unitaria alrededor de lo socio-ambiental en Chile, sí se puede señalar la multiplicación creciente del descontento.

Por último, cabe señalar algunos limitantes del estudio de los conflictos y protestas socioambientales a partir de bases de datos. Uno de los rasgos comunes entre estas distintas iniciativas de mapear y categorizar los conflictos es el hecho de caracterizar el conflicto a través de un elemento visible -aparición en medios de comunicación $(\mathrm{OdC})$ o manifestación pública de visiones distintas (INDH y EJAtlas) - o la existencia de una organización o la realización efectiva de acciones colectivas -OCMAL, OLCA y EJAtlas. Así, más allá de una confusión posible entre conflicto y protesta, hay una tendencia a reducir analíticamente el conflicto a la movilización y esto constituye un limitante importante del estudio. Otras literaturas, como los trabajos de Scott sobre resistencias populares (1985) o de Auyero \& Swistun (2007) sobre sufrimiento ambiental, ponen de relieve las distintas formas de expresar un descontento, las dificultades en formular un malestar ligado a temas ambientales y las profundas desigualdades que existen en las capacidades que tienen los actores en movilizarse y construir un repertorio de acción común.

Tomando en cuenta estas últimas aproximaciones, y considerando el objetivo de abarcar y registrar los conflictos socioambientales en el país, nos parece necesario combinar los análisis de bases de datos con otras metodologías de investigación. Tal como lo recuerdan Aliste y Stamm (2016), en la RM no siempre los problemas ambientales pueden concretarse en conflictos, pues "importantes sectores de la ciudad viven expuestos a condiciones de calidad ambiental deplorables (...) sin que exista por parte de la población una sensación de detrimento o reclamo por estas condiciones que en muchos casos pueden, incluso, ser consideradas como "normales" (Aliste \& Stamm, 2016, p. 58). Las situaciones de zonas de sacrificio como la de Puchuncaví Quintero, que combinan sufrimiento ambiental con una profunda desigualdad social, nos obligan a repensar nuestras formas de analizar el conflicto socio-ambiental en Chile y a seguir adelante con esta agenda de investigación.

\section{REFERENCIAS}

Aliste, E., \& Stamm, C. (2016). Hacia una geografía de los conflictos socioambientales en Santiago de Chile: Lecturas para una ecología política del territorio. Revista de Estudios sociales, 35(55), 45-62. doi:10.7440/res55.2016.03
Arriagada, E., (2012). El conflicto por polimetales en Arica. Movilización social, desarticulación local e intervención centralizada. En G. Delamaza, N. Cunill, \& A. Joignant, (Eds.), Nueva agenda de descentralización en Chile. Sentando más 
Allain, M. (2019). Conflictos y protestas socioambientales en Chile: Reflexiones metodológicas y resultados. Revista de Sociología, 34(1), 81-101. doi: 10.5354/0719-529X.2019.54271

actores a la mesa (pp. 459-485). Santiago de Chile: Ril Editores.

Auyero, J. \& Swistun, D. (2007). Expuestos y confundidos. Un relato etnográfico sobre sufrimiento ambiental, Iconos. Revista de Ciencias Sociales, 28, 137-152. doi: 10.17141/iconos.28.2007.216

Beuf, A. (2017). El concepto de territorio: De las ambigüedades semánticas a las tensiones sociales y políticas. En A. Beuf \& P. Rincón (Eds), Ordenar los territorios. Perspectivas críticas desde América Latina (pp. 3-23). Bogotá: Universidad de los Andes, Universidad Nacional de Colombia, Instituto Francés de Estudios Andinos,

Biskupovic, C. \& Stamm, C. (2016). Quand les mobilisations citoyennes transforment la précordillère des Andes:Le cas d'une association de riverains à Santiago du Chili, Revue de Géographie Alpine, 104 (1), 1-19. doi: 10.4000/rga.3204

Bourdieu, P. (2002). Questions de sociologie. Paris: Les Éditions de Minuit.

Calderón, F. (2012). La protesta social en América Latina. Buenos Aires :Siglo Veintiuno Editores

Carruthers, D. \& Rodriguez, P. (2009). Mapuche protest, environmental conflict and social movement linkage in Chile", Third World Quarterly, 30(4), 743-760. doi: $10.1080 / 01436590902867193$

Carruthers, D. (2001). Environmental politics in Chile: Legacies of dictatorship and democracy. Third World Quarterly, 22(3), 343-358. doi :10.1080/01436590120061642

Centro de Estudios de Conflicto y Cohesión Social. (2018). Informe Anual Obsrvatorio de Conflictos $2018 . \quad$ Disponible en https://www.coes.cl/wpcontent/uploads/2018/11/ORG_InformeObservatorio-de-Conflictos-2018_5nov.pdf

Centro de Estudios de Conflicto y Cohesión Social. (2017). Notas de Política Pública $n^{\circ} 4$, conflicto social en Chile 2015-2016: disputando mitos. Santiago de Chile: COES
Delamaza, G., Maillet, A., Martínez, C.(2017). Socio-Territorial Conflicts in Chile: Configuration and Politicization (20052014), ERLACS, 104, 23-46. doi: 10.18352/erlacs. 10173

Di Méo, G. (1998). Géographie sociale et territoires. Paris: Nathan.

Fillieule, O. (2007). On n'y voit rien: Le recours aux sources de presse pour l'analyse des mobilisations protestataires. En P. Favre (Ed), L'atelier du politiste: Théories, actions, représentations (pp. 215-240). Paris: La Découverte.

Fundación Relaves. (s.f.). Casos : El soldado y Las palmas. Disponible en http://www.relaves.org/

Garretón, M. (2012). Neoliberalismo corregido y progresismo limitado: Los gobiernos de la concertación en Chile, 1990-2010. Santiago de Chile: Editorial ARCIS-CLACSOPROSPAL.

Instituto Nacional de Derechos Humanos. (2018). Censo de población y viviendas 2017. Santiago: Instituto Nacional de Estadística. Disponible en http://www.censo2017.cl/descargue-aquiresultados-de-comunas/

Instituto Nacional de Derechos Humanos. (2012). Mapa de conflictos socio ambientales en Chile. Disponible en https://mapaconflictos.indh.cl/\#/

Instituto Nacional de Derechos Humanos. (2018). Informe misión de observación a comuna de Til Til, Provincia de chacabuco, Región Metropolitana, Octubre 2017 febrero 2018. Disponible en https://bibliotecadigital.indh.cl/bitstream/ha ndle/123456789/1149/misiontiltil.pdf?sequence $=1$

Joignant, A., Morales, M. \& Fuentes, C. (2016). Malaise in representation in Latin American countries. New York: Springer

Neveu, É. (2015). Sociologie politique des problèmes publics. Paris: Armand Colin. 
Allain, M. (2019). Conflictos y protestas socioambientales en Chile: Reflexiones metodológicas y resultados. Revista de Sociología, 34(1), 81-101. doi: 10.5354/0719-529X.2019.54271

Maillet, A. \& Albala, A. (2018). Conflictos socioambientales en los proyectos eléctricos en Chile (2005-2016): Un análisis configuracional. América Latina Hoy, 79, 125-149. http://dx.doi.org/10.14201/alh201879125149

Maillet, A, \& Delamaza, G. (2017). Protestas en torno a conflictos extractivos en Chile (20152016), algunas tendencias generales y la especificidad del sector forestal. En COES (Ed.), Notas de Política Pública $n^{\circ} 4$, conflicto social en Chile 2015-2016: disputando mitos (pp.38-45). Santiago de Chile: COES

Martinez Neira, C. \& Delamaza, G. (2018). Coaliciones interétnicas, framing $\mathrm{y}$ estrategias de movilización contra centrales hidroeléctricas en Chile: ¿Qué podemos aprender de los casos de Ralco y Neltume?. Middle atlantic review of Latin American Studies, 2(1), 68-96. doi: 10.23870/marlas. 180

Observatorio de Conflictos Mineros de América Latina. (s.f.). OCMAL:¿Quiénes somos?. Disponible en https://www.ocmal.org/ocmal/\#que-hacemos

Observatorio Latinoamericano de Conflictos Ambientales. (s.f.).OLCA: Comunidades en acción. Disponible en http://olca.cl/oca/index.htm

Romero H., Castro, F., Jaramillo, N., \& Alister, C. (2017). Conflictividades en la Araucanía y más allá. En COES (Ed.), Notas de Política Pública $n^{\circ} 4$, conflicto social en Chile 20152016: disputando mitos (pp.38-45). Santiago de Chile: COES

Tironi, M. \& Zenteno, J. (2012). Licencia social para operar. Sostenibilidad y las justificaciones de la RSE en la gran minería chilena. En J. Ossandón, \& E. Tironi (Eds.), Adaptación. La empresa chilena después de Friedman (pp.315-344). Santiago: Ediciones UDP.

Temper, L., Del Bene, D., \& Martinez-Alier. J. (2015). Mapping the frontiers and front lines of global environmental justice: The EJAtlas. Journal of Political Ecology, 22, 255-278. doi: 10.2458/v22i1.21108

Schaeffer, C. (2016). Democratizing the Flows of Democracy: Patagonia sin represas in the awakening of Chile's civil society. En S. Donoso \& M. von Bülow (Eds.), Social movements in Chile. Organization, trajectories, and political consequences (pp. 131-161). New York: Palgrave-MacMillan.

Sepúlveda, C. \& Villarroel, P. (2012). Swans, conflicts, and resonance: Local movements and the reform of Chilean Environmental Institutions. Latin American Perspectives, 39(4), 181-200. doi: 10.1177\%2F0094582X12441519

Scott, J. (1985). Weapons of the weak. Everyday forms of peasant resistance, Yale University Press: New Haven and London.

Valenzuela, E., Penaglia, F. \& Basaure, L. (2016). Acciones colectivas territoriales en Chile, 2011-2013: De lo ambientalreivindicativo al autonomismo regionalista. EURE (Santiago), 42(125), 225-250. doi: http://dx.doi.org/10.4067/S025071612016000100010

Manuscrito recibido: 11-07-2019 Manuscrito aceptado: 19-07-2019 